\title{
Discovery of the Potential Role of Sensors in a Personal Emergency Response System: What Can We Learn from a Single Workshop?
}

\author{
Femke De Backere*, Stijn Verstichel*, Jan Van den Bergh ${ }^{\dagger}$, Shirley A. Elprama ${ }^{\ddagger}$, Femke Ongenae*, \\ Filip De Turck* ${ }^{*}$ An Jacobs ${ }^{\ddagger}$ and Karin Coninx ${ }^{\dagger}$ \\ ${ }^{*}$ Dept. of Information Technology (INTEC) - Ghent University - iMinds \\ G. Crommenlaan 8/201, 9050 Ghent, Belgium \\ Email: firstname.lastname@intec.UGent.be \\ ${ }^{\dagger}$ Hasselt University - tUL - iMinds \\ Expertise Centre for Digital Media, Wetenschapspark 2, Diepenbeek, Belgium \\ E-mail: firstname.lastname@uhasselt.be \\ $\ddagger$ VUB - SMIT - iMinds \\ Pleinlaan 9, 1050 Brussels, Belgium \\ Email: firstname.lastname@vub.ac.be
}

\begin{abstract}
Capturing knowledge from domain experts is important to effectively integrate novel technological support in existing care processes.

In this paper, we present our experiences in using a specific type of workshop, which we identified as a decision-tree workshop, to determine the process and information exchange during the usage of a Personal Emergency Response System (PERS). We conducted the workshop with potential current and future users of a PERS system to investigate the potential of contextand social awareness for such a system. We discuss the workshop format as well as the results and reflection on this workshop.
\end{abstract}

\section{INTRODUCTION}

Fall incidents can have a major impact on the life of older adults. It can lead to a degradation of autonomy or even the admission of the older adult in a retirement home. Older adults at risk can use a Personal Emergency Response System (PERS) [1], which enables them to press a button when they are in need of help like when a fall occurred. When triggering an alarm, a call center is notified. The operator will then contact a friend, relative or neighbour from the predefined list to notify that an incident took place and that somebody should assist the fall victim. However, the current implementation of the process is static and the prevailing context is not taken into account. For example, the list of persons to be contacted has a fixed order and the person's availability to help the fall victim is unknown.

By taking pervasive and context-aware information and data into account [2], [3], it is possible to make the PERS system and the incident handling process more flexible. This also would improve the quality of care and moreover the quality of life of older adults. However, to make the PERS system more flexible, more specific the caregiver selection algorithm and the process of handling a fall, domain knowledge of the involved stakeholders has to be revealed. The stakeholders involved in this process are the formal caregivers, such as the desktop operator (always a nurse), who receives the emergency call (at the home care organisation), nurses providing care to older adults, the persons that are contacted in case of an emergency, which could be family, neighbours, friends, and others and the older adults themselves.

In this paper, we discuss one workshop we organized to gain insights in the desired process and the domain knowledge used within it and present lessons learned with respect to the applicability and organisation of the specific type of workshop.

\section{RELATED WORK}

Several methodologies exist to capture domain knowledge[4]. This methdologies where analyzed and the most adequate one was chosen, which enables an optimization of the PERS system:

- The INSIGHT methodology and tool makes it possible for the domain expert to make the knowledge more consistent. This approach has been used in an Intensive Care Unit (ICU) [5].

- Living Lab methodologies are a research concept, in which a user-centered, open-innovation ecosystem is established. This way research and innovation processes can be tested and evaluated. A few examples of living labs are the Care Living Labs in Belgium ${ }^{1}$ and the iLab.o of iMinds ${ }^{2}$ [6].

- Tools and applications such as MindTool [7]

- Interviews, document collection, and observations can also be used to capture the knowledge within a certain organization or from people [8].

- Within the Innovation Binder approach, the balance is found between social and technical development [9]. This

\footnotetext{
${ }^{1}$ www.zorgproeftuinen.be

${ }^{2}$ www.openlivinglabs.eu/livinglab/iminds-ilabo
} 
interdisciplinary procedure can be used to confront multiple viewpoints from user/social, technology and business perspective, to make choices more explicit and coordinate the team to work together to a common abstract goal. This way, both domain experts and researchers with a social or technical background can share their expertise and knowledge.

- Ontologies [10] are constructed to formally capture the knowledge within a specific domain. WebProtégé is a lightweight ontology editor and knowledge acquisition tool tailored for domain experts [11].

Several methods can be used to detect the domain knowledge, necessary to construct an applicable ontology. The ontology co-design method [12] is one of these methods. Decision tree workshops are one of the used steps in defining an ontology, more specifically, how to capture the decision processes within a domain. This codesign methodology can be part of the Innovation Binder approach.

Previous research by the authors [12] has revealed that traditional knowledge capturing techniques, such as stakeholder questionnaires and observations do not always reveal all necessary details to sufficiently understand the problem statement and underlying algorithmic dependencies and to design an information system that end-users really want to embrace.

As the goal of this research is to capture the knowledge of the domain experts and stakeholders about how a fall should be handled when the PERS system is activated, some considerations should be taken into account:

- Inital user research has taken place, but there are some gaps within the existing guidelines that need to be filled.

- The stakeholders, as discussed in Section I, should feel at ease and the used approach should be easy to understand.

- The results of this research will be integrated in the developed FallRisk system [3] ${ }^{3}$, where ontologies are used to describe the domain of continuous care and fall handling.

Therefor, the choice was made to use the decision tree workshop approach as described in the ontology co-design method.

\section{WORKSHOP}

The following subsections will discuss the preparation, the objectives, the participants and the methods used within the workshop.

\section{A. Preparation}

In order to result in a successful experience for all participants, it is important that the workshop is well prepared and focussed on one or more specific scenarios. In the case of fall handling and PERS, a number of characteristic and recognisable situations has been defined beforehand. In this case the scenario was used where an older adult fell.

\footnotetext{
${ }^{3}$ http://www.iminds.be/en/projects/2014/03/19/fallrisk
}

\section{B. Objectives}

An important goal of this workshop was the translation of the conceptual model into a formal one. This can be done through the definition of axioms which restrict the interpretation of the information in a given situation, e.g. when a fall incident needs to be treated as urgent and/or critical. Most of these restrictions were derived from the information obtained during the observations. The general goal of this type of workshop, as described in [12] is to capture these decision processes, which can then be translated into additional ontology concepts and rules/axioms.

In contrast to these general objectives, the main goal of this specific workshop at hand was not primarily to discover all logic and necessary information for the decision making processes and workflows, but rather to check whether the existing guidelines really do reflect current day practices and requirements from all stakeholders. Of course, any missing information in the current guidelines can then be completed using the feedback from the participants during the workshop.

\section{Participants}

Five participants were recruited via a home care organization based on their different roles and tasks: one older adult using the PERS system (F, 86), an informal caregiver (and also contact person) of the older adult (her daughter, 65) and three employees (of which two were a nurse) working at the home care organisation. Both nurses worked only part time as a home care nurse, while one (F, 35) worked part time as an operator at the call center answering calls from the PERS and the other $(\mathrm{F}, 54)$ was responsible for installing equipment such as PERS but also special beds or matresses. The third employee (F, 29) was not a nurse, but was also responsible for installing equipment. The workshop lasted about 1.5 hours. Three researchers were present to lead the workshop.

\section{Methods}

[12] gives a detailed description of all methods used in decision tree workshop. However, for the convenience of the reader, a summary is presented.

First, the participants were asked to write down a number of situations they have come across and would like to see improved by the new PERS procedure. Then, during the main part of the workshop, they were asked to impersonate the actual intelligent all-knowing system. The aforementioned situations were selected to further discuss how this intelligent PERS should ideally handle the situation.

Each situation started with a very limited initial setup and was visualised on an easily understandable visualisation, in this case a city map, post-its and pawns, as shown in Figure 1. To make a sensible decision, the participants playing the role of the system, could ask for additional information about the situation by asking questions, e.g., 'Do we know the personal details of the person who has fallen?', 'Do we know who can be contacted?', 'What are their roles?'. Instead of immediately giving an answer, a discussion was encouraged about the importance of the requested information by asking 


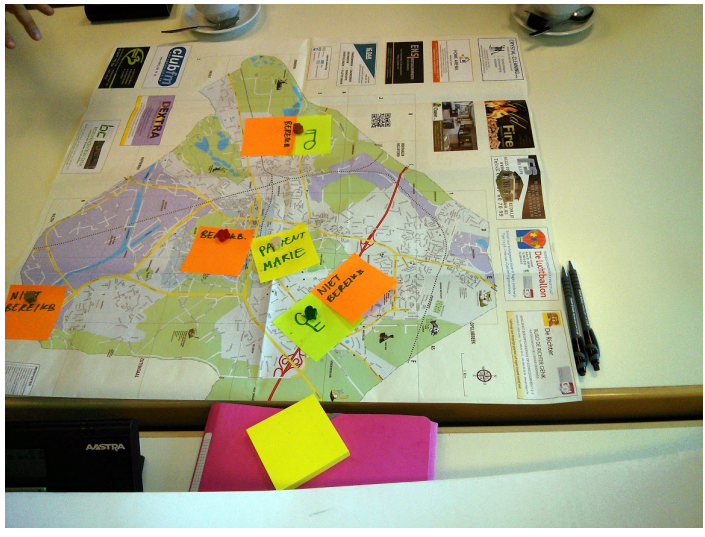

Fig. 1. Materials used to sketch the context of a PERS call

three questions: (1) 'Why do you feel the answer to this question is pertinent?' (2) 'Does everyone agree?' (3) 'Can you give examples of answers to this question?'. Finally, the question was answered and visualized. Further questions unravel the situation. The questions and the order in which they were asked, give the researchers insights into the needed information and its importance for making a decision. The ontology engineer processed the outcome on paper in the form of a decision tree. The outcomes described in Section IV are based on watching and listening the recorded footage of the workshop and the decision tree that was drawn during the workshop.

\section{OUtComes}

\section{A. Process}

At the start of the workshop, the participants were asked to write down a situation or experience they encountered with PERS. The nursing home employees seemed to understand the assignment immediately, while the informal caregiver was a bit reluctant to participate and had difficulties to write down a situation. Based on the response of the older adult, it was not clear if she understood the assignment, but she was able to tell about a situation in which she fell. One researcher worked together with the older adult to write down a particular situation, but she sometimes relied on her daughter to fill in some details, for instance, whether she already had the PERS at the moment of that particular fall.

When discussing which questions should be asked first, all participants contributed, but some were more dominantly present than others. In the beginning, it had to be repeated and clarified that the questions were not asked to the patient, but to the all-knowing system. Especially the nurses - who took more actively part in the discussion - had to be reminded repeatedly that the exercise was about the desired situation, which might be different from their current working procedures. When the participating older adult made remarks, it was often related to incidents that happened to herself.

\section{B. Results}

The workshop confirmed the wanted procedure largely corresponds to the process currently followed when a PERS call is received. A simplified version of this process is:

1) determine whether it is a false alarm, if so, close call and $\log$ this information

2) determine whether there is a need to immediately call an ambulance (heavy bleeding, immobility of arms or legs, no answer, which might indicate unconsciousness)

3) determine location of the caller

4) if fall victim is in house and key may be necessary, find someone that can provide entrance to the house

5) follow up on caregiver presence and care giving (minimally 1 update every 15 minutes)

6) if person present cannot provide care find additional person that can provide care

7) follow up on caregiver presence (minimally 1 update every 15 minutes)

8) close call and log provided care

The workshop however also resulted in qualitative insights; experiences of the persons participating in the workshop and possible opportunities where sensor technology can help without disrupting the overall process.

- Availability of caregivers is a crucial factor, for which a manual procedure exists to at least take into account long periods of absence (vacation). Experience from nurses working in the call center learned that this information is not always provided. Technology could provide further improvements through the use of digital agendas, from which availability information can be derived. Automated reminders can help caregivers to keep their availability up-to-date.

- Time to get to the person, from which the call originates is crucial. Currently, the contact persons actual location is unknown, potentially losing time since calls are made to people who are currently far from the fall victim. Availability of automatically determined location can be beneficial as they give access to the actual location. Contacts can augment this information to determine likely actual time of arrival. An important, but very difficult balance has to be found here. On the one hand, real-time and up-to-date location information of the caregivers can be very beneficial, but on the other hand personal privacy can potentially be in danger if too much information is gathered and certainly when it is not properly managed.

- Location of the patient or door sensor information, in cases where a key would be needed, can be valuable especially when contacts with a key are not available. According to the nurses, it often happened that people who were available to help the fall victim did not have key access to the fall victim's house.

- Also, it was evident that in the current working procedures, there was sometimes a 'struggle' between the wishes of the nurses and the PERS users. For instance, the older adults wanted to be contacted by a specific person 
(for instance due to fights with other contact persons), while the nurses thought it was more important that the person would be contacted who is closest in distance to the fall victim. However, because the nurses respect the wishes of the PERS users, the preferred contact person is contacted first. For instance, if they ask to call an ambulance, the nurse has to call an ambulance.

\section{Discussion}

\section{A. Workshop outcomes}

The workshop was successful in gathering which information is needed during the process of PERS. Due to the nature of the workshop (an all knowing being), we also learned when and why this information is required, but not how this information is obtained. The natural tendency of the involved users to base themselves on the existing process can be used to identify parts of which acceptance of a new solution may be difficult and may thus be used at a later point to ease migration to a new process.

The workshop also provided insights about the diversity of priorities across stakeholders and how these are weighted to reach an agreement. The role of the PERS is to assist in the process, not to replace the final human decision maker.

In contrast to previous experience, the decision tree workshop was organised independently from all other knowledge gathering tasks. One important reason for this is that the ontology models, which have been engineered over time previously, could be easily incorporated and minimally enhanced for the specific domain at hand. Restricting the interpretation of the concepts in the ontology, by means of axioms, was a part of the domain knowledge that had to be engineered for the specific setting of a PERS. A decision tree workshop has proven to be the ideal tool to accomplish this task.

\section{B. Stakeholder involvement}

A decision tree workshop is an opportunity for discussion with relevant stakeholders. However, the focus of the workshop was a specific process that mainly involved nurses working at the call center (only $2 / 5$ participants). As a consequence, the other participants had less to add to the workshop and the moderator had to pay attention to address the other participants to involve them in the workshop. Note that the format of the workshop facilitates this, an agreement is necessary and thus the moderator can explicitly involve all stakeholders.

The older adult, attending the workshop, required some extra help from one researcher in completing the assignment. Furthermore, it was unclear if she understood the purpose of the workshop. The concept of the all-knowing machine was perhaps too abstract. As with most workshops, the role of the moderator is important to maintain focus and encouraging all participants to express themselves.

\section{Organisation and preparation}

It is important for a decision tree workshop to idenyify and invite relevant stakeholders, in our case nurses, working at the call center, an older adult and an informal caregiver/contact person and a nursing home employee, responsible for installing PERS. During the workshop, three researchers were present (one for visualizing the decision tree, two for asking the questions and moderating the workshop). We recommend to practice the workshop in advance with volunteers, because it helps in the preparation and understanding of the workshop concept, especially for researchers new to the method.

When deciding what information was needed about the contact persons, we used a map of a city in Belgium to visualize the locations of the informal caregivers compared to the fall victim. This ensured the same mental model of the proximity of the contact persons (visualized as pawns), and in addition, post-its were used to indicate whether these contact persons had key access to the fall victims home.

\section{ACKNOWLEDGMENT}

The iMinds FallRisk project is cofunded by iMinds (Interdisciplinary Institute for Technology), a research institute founded by the Flemish Government. Companies and organizations involved in the project are COMmeto, Televic Healthcare, TP Vision, Verhaert and Wit-Gele Kruis Limburg, with project support of IWT.

\section{REFERENCES}

[1] V. Hessels, G. S. Le Prell, and W. C. Mann, "Advances in personal emergency response and detection systems," Assistive Technology, vol. 23, no. 3, pp. 152-161, 2011.

[2] H. Viswanathan, B. Chen, and D. Pompili, "Research challenges in computation, communication, and context awareness for ubiquitous healthcare," Communications Magazine, IEEE, vol. 50, no. 5, pp. 92-99, 2012.

[3] F. De Backere, F. Ongenae, F. Van den Abeele, J. Nelis, P. Bonte, E. Clement, M. Philpott, J. Hoebeke, S. Verstichel, A. Ackaert et al., "Towards a social and context-aware multi-sensor fall detection and risk assessment platform," Computers in biology and medicine, 2014.

[4] B. R. Gaines, "Knowledge acquisition: Past, present and future," International Journal of Human-Computer Studies, vol. 71, no. 2, pp. 135-156, 2013.

[5] D. Sleeman, S. Rogers, L. Moss, A. Aitken, and J. Kinsella, "Insight: Helping domain experts make their knowledge more consistent," on Capturing and Refining Knowledge in the Medical Domain (K-MED 2012), p. 22, 2012.

[6] E. Almirall, M. Lee, and J. Wareham, "Mapping living labs in the landscape of innovation methodologies," Technology Innovation Management Review, vol. 2, no. 9, 2012.

[7] G.-J. Hwang, H.-C. Chu, Y.-S. Lin, and C.-C. Tsai, "A knowledge acquisition approach to developing mindtools for organizing and sharing differentiating knowledge in a ubiquitous learning environment," Computers \& Education, vol. 57, no. 1, pp. 1368-1377, 2011.

[8] M. Wilkesmann and U. Wilkesmann, "Knowledge transfer as interaction between experts and novices supported by technology," Vine, vol. 41, no. 2, pp. 96-112, 2011.

[9] A. Jacobs, P. Duysburgh, F. Ongenae, A. Ackaert, L. Bleumers, and S. Verstichel, "The Innovation Binder Approach: A Guide Towards a Social-Technical Balanced Pervasive Health System," in Pervasive Health, A. Holzinger, M. Ziefle, and C. Röcker, Eds. Springer London, 2014, pp. 69-99.

[10] T. R. Gruber, "A translation approach to portable ontology specifications," Knowledge acquisition, vol. 5, no. 2, pp. 199-220, 1993.

[11] T. Tudorache, C. Nyulas, N. F. Noy, and M. A. Musen, "Webprotégé: A collaborative ontology editor and knowledge acquisition tool for the web," Semantic web, vol. 4, no. 1, pp. 89-99, 2013.

[12] F. Ongenae, P. Duysburgh, N. Sulmon, M. Verstraete, L. Bleumers, S. De Zutter, S. Verstichel, A. Ackaert, A. Jacobs, and F. De Turck, "An ontology co-design method for the co-creation of a continuous care ontology," APPLIED ONTOLOGY, vol. 9, no. 1, pp. 27-64, 2014. [Online]. Available: http://dx.doi.org/10.3233/AO-140131 